\title{
A Cross-Domain Trust Model for Reputation Management in Hybrid CDNi-P2P Network
}

\author{
Shi Li, Inshil Doh, Kijoon Chae ${ }^{+}$ \\ Department of Computer Science and Engineering, Ewha Womans University, Seoul, Korea
}

\begin{abstract}
Content delivery network (CDN) as a distributed network architecture enhances efficient delivery of contents. And the interconnection of different CDNs (CDNi) improves the efficiency and the experience of end users. As another distributed network with high availability and high performance, Peer-to-peer (P2P) network can provide an efficient way for resource sharing. To combine the advantages of the two networks, in this paper, we propose a hybrid CDNi-P2P architecture and trust management models to achieve more efficient delivery of contents. In general, different systems adopt different reputation evaluation standards. This leads to the disparate trust values for the mobile users in different systems. Based on the architecture, we propose two trust models to solve this problem: the local trust model and the cross-domain trust model. Based on the proposed trust models, the mobile user can transform his local trust to the mobile trust in the new domain. And the mobile trust is converted to the new trust and then used in the new domain. We can avoid the disparate trust values of the same user in different domains and improve the availability of the contents possessed by the mobile users during the user moving among different domains.
\end{abstract}

Keywords: CDNi, P2P, trust, reputation, mobile, cross domain.

\section{Introduction}

Nowadays, there are two major technologies for large scale video streaming over the Internet: Content Delivery Networks (CDNs) and Peer-to-Peer (P2P) networks. Both of them can distribute contents with high availability and high performance. With CDNs, content is distributed to cache servers (edge servers) located close to end users by origin server, resulting in fast, reliable applications and Web services for the users [1]. There are many commercial CDN companies, such as Akamai, AT\&T, NTT Communication, Limelight, Mirror Image, Level 3, etc. In fact, it is extremely expensive to deploy and maintain CDN servers. For this reason, CDN architectures do not benefit from high scalability. However, P2P networks can be highly scalable because of its low start-up cost and peers instead of dedicated and expensive servers. The complementary advantages of CDN and P2P networks lead to the combination of them as a hybrid CDN-P2P architecture and create a distribution system with higher scalability and reliability [2], [3].

Content Delivery Network interconnection (CDNi) as a new interactive network infrastructure allows the information and contents to be transmitted between different CDNs through specific interfaces. CDNi provides all of the benefits of CDN and also has some unique characteristics itself. In CDNi, end users do not need to register at all CDN providers to obtain the contents from different Content Service Providers (CSPs). When the content is not cached in any edge server of the CDN registered, end-user's request will be redirected to other CDNs to recapture the content through the interfaces between different CDNs (CDNi requires the specification of interfaces and mechanisms to address issues such as request routing, distribution metadata exchange, and logging information exchange across CDNs [4]). As a result, the content could be delivered through a "CDN chain" and transmitted to end users by the closest CDN. Here, there are two categories of

\footnotetext{
+ Corresponding author. Tel.: +82-2-3277-2370; fax: +82-2-3277-3506.

E-mail address: kjchae@ewha.ac.kr.
} 
CDNs: the one who caches contents from CSP is called upstream CDN ( $\mathrm{uCDN}$ ), and the one who delivers contents directly to the end user is called downstream CDN (dCDN). In order to deliver contents more efficiently, in this paper, we combine CDNi and P2P architectures as a hybrid CDNi-P2P network, which can combine the advantages of $\mathrm{CDNi}$ and $\mathrm{P} 2 \mathrm{P}$ networks. Based on the proposed hybrid CDNi-P2P architecture, end-users could receive the contents from the closest edger server of the $\mathrm{dCDN}$ which who registered, as well as other peers which locate in the same domain.

In hybrid CDNi-P2P network, all the end users as peers are both consumers and providers. When an end user requests content from other peers, some might be honest and provide accurate content which he received before from the edge server of dCDN, others might be self-serving and unwilling to provide content for other peers, and the others might be even malicious by providing false content or harming the consumers [5]. Trust model provides a way to generate trust based on the historical behaviour of a peer [6]-[10]. The larger a peer's trust value is, the higher probability the accurate content could be provided. Meanwhile, there are some mobile peers which move among different systems or domains, from the reputation estimated point of view, they need the respective trust values in each P2P system. In general, different P2P systems and domains adopt different trust models and reputation evaluation standards. This will lead to the disparate trust values for the same peer in different domains, even if it always has the same performance. However, according to the existing reputation systems, most researches focus on the trust model for the same systems or domains. Trust model among different domains has never been considered. Thus in this paper, we will propose a cross-domain trust model for the hybrid CDNi-P2P network.

The rest of this paper is organized as follows: the local trust model will be introduced in Section 2, and the hybrid CDNi-P2P architecture and the domain-cross trust model will be proposed in Section 3, and the Section 4 concludes this paper.

\section{Proposed Local Trust Model}

As a preparation to propose the cross-domain trust model, there should be a relative reputation evaluation for each participant in a local system, which can indicate the ranking of the reliability of each individual participant. In this section, we propose a local trust model to generate the relative reputation value for each participant according to the different reputation evaluation standards used by the local system.

\subsection{Background}

According to the existing online reputation systems and researches, there are mainly two approaches to evaluate the reputation of a participant for a specific network. In the first approach, the ratings of both service receiver and provider are given by a bi-directional or one-directional rating way after each transaction. The form of ratings could be reputation scores, feedback ratings, and positive feedback rate et al. And the overall reputation of a participant is the sum of these ratings which is called trust value denoted by $v$. The examples of online reputation systems by using this approach are online auction system eBay [11], Amazon [12] and Taobao [13]. Generally, the trust value is an integer equal or greater than zero which is a public value to all the participants in the system. And other participants can decide their trust in a participant based on this trust value. In the second approach, both service receiver and provider can also be rated by each other after the transaction, and they calculate the trust degree to the others [6]-[10]. The trust degree is a value between 0 and 1 which can be denoted by $d$. For example, when there are two participants $i$ and $j$, as mentioned above, $i$ could give rating to $j$ after each transaction, and the trust degree of $i$ trusting in $j$ denoted by $d_{i j}$ could be the ratio of positive ratings.

\subsection{Local Trust Model}

The objective of local trust model is to calculate the relative reputation degree of each participant in a local system. We call it local trust degree which should be a value between 0 and 1 . According to the two approaches of reputation system mentioned above, we will propose two methods to calculate the local trust degree of an individual participant respectively. Fig. 1 illustrates the two types of reputation system: the trust value reputation system and trust degree reputation system. 


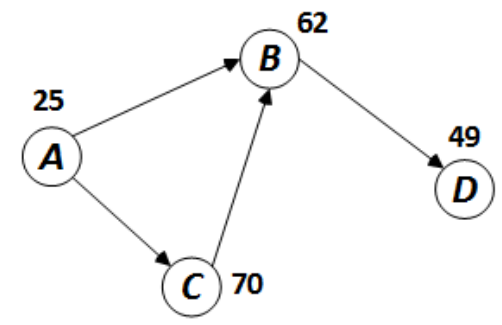

(a)

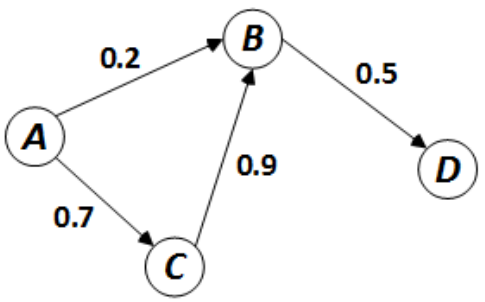

(b)

Fig. 1: (a) "Trust value" reputation system (b) "Trust degree" reputation system.

\section{“Trust value” reputation system}

We assume that there are $n$ participants in the system, and each participant $i, i \in(1, n)$, has a trust value $v_{i}$ which is given by the other participants after the transaction. Thus, the local trust degree of $i$ can be calculated as follows.

$$
\text { local_d } d_{i}=\frac{v_{i}}{\max _{j \in(1, n)} v_{j}}
$$

Obviously, local_ $d_{i}$ is a value between 0 and 1 , which indicates the ranking of $i$ 's trust value in the system. As an example shown in Fig. 1 (a), the local trust degree of $D$ local_ $d_{D}$ is 0.7 according to the equation (1).

\section{“Trust degree" reputation system}

In order to calculate the local trust degree of participant $j$, firstly, we need to obtain the trust degrees of all the other participants trusting in $j$, i.e., we need all the $d_{i j}, i \in(1, n)$, which can be calculated as follows.

$$
d_{i j}=\sum_{k=1}^{n} d_{i k} d_{k j}
$$

Equation (2) is a recursive equation, because the equation is used recursively in computing $d_{i k}$ or $d_{k j}$, it is also possible to use this equation. As an example shown in Fig. 1 (b), $d_{A D}$ can be calculated by $d_{A B} \times d_{B D}+$ $d_{A C} \times d_{C D}$, and here $d_{C D}$ can also be calculated by $d_{C B} \times d_{B D}$, i.e., $d_{A D}=d_{A B} \times d_{B D}+d_{A C} \times d_{C B} \times d_{B D}=d_{A B} \times d_{B D}+$ $d_{A B} \times d_{B D}$. It means that there are two ways to calculate $d_{A B}$ : one is the direct trust degree and the other is the indirect trust degree. In this case, we will use the direct trust degree only to calculate $d_{A D}$, whose result is 0.1 . Similarly, we can also calculate the $d_{B D}$ and $d_{C D}$ as 0.5 and 0.45 respectively.

According to the trust degrees of $j$ given by all the other participants in the system, the local trust degree of participant $j$ can be calculate as follows.

$$
\text { local_d } d_{j}=\frac{\sum_{i=1}^{n} d_{i j}}{n}
$$

Here, local_ $d_{j}$ is a value between 0 and 1 , which can indicate the average ranking of the trust of $j$ based on the ratings given by all the other participants in the system. Thus, as the example in Fig. 1 (b), the result of local_d $d_{D}$ is 0.35 based on the equation (3).

\section{Proposed Cross-Domain Trust Model for Hybrid CDNi-P2P Network}

For the mobile peers which move among different systems or domains, from the reputation estimated point of view, they need the respective trust values in each P2P system. In general, different P2P systems or domains adopt different trust models and reputation evaluation standards. This will lead to the disparate trust values of the same peer in different domains, even if it always has the same performance. In this section, we will propose a cross-domain trust model for the mobile peer in hybrid CDNi-P2P network.

\subsection{Hybrid CDNi-P2P Network Architecture}

Proposed hybrid CDNi-P2P network architecture is shown in Fig. 2. In the architecture, there are two types of CDNs: $\mathrm{uCDN}$ and dCDN. Contents provided by CSP are only stored in the edge servers of uCDN. If an end user $u_{l}$ who can only obtain service directly from dCDN-A sends a content request to origin server, the content 
will be delivered from uCDN to dCDN-A and then transmitted to end user through the closet edge server of dCDN-A. If there is another end user from the same domain also wanting to obtain this content, he can get the content directly from $u_{l}$ as well. And here, each CDN can play the role as uCDN and dCDN together at the same time, just based on the content requested by the end user.

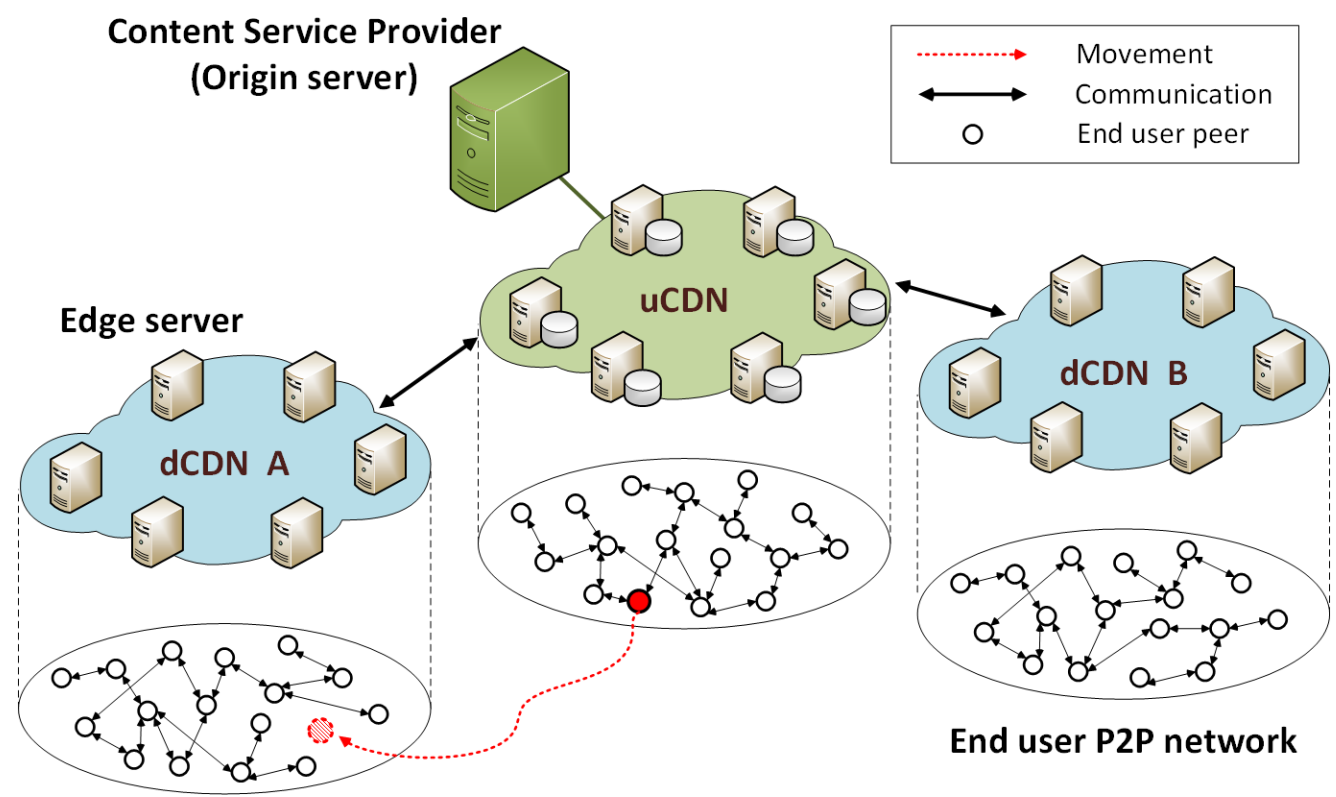

Fig. 2: Hybrid CDNi-P2P network architecture.

\subsection{Cross-Domain Trust Model}

In Fig. 2, there is a mobile end user peer (the red solid point) who can move between uCDN and dCDN domains. For some mobile peers who have high trust values in one P2P domain, their trust values may be initialized based on the trust model used in the new domain, when they move to another domain, because they are new comers. This will lead to the waste of resources, because other peers are not willing to obtain content from a new peer. In addition, they need to take a long time to accumulate trust in the new domain.

We assume that the uCDN and dCDN can also rate each other after each content delivering between them, and that the evaluation method is based on the trust degree reputation system as mentioned in Section 2. The two CDNs are denoted by $C D N_{x}$ and $C D N_{y}$ respectively. The trust degree that $C D N_{x}$ rates $C D N_{y}$ is $d_{x y}^{C D N}$, and the trust degree that $C D N_{y}$ rates $C D N_{x}$ is $d_{y x}^{C D N}$. If a mobile end user $u_{m}$ in $C D N_{x}$ domain with local trust degree local_ $d_{u_{m}}^{C D N_{x}}$ (as mentioned in Section 2) is willing to move to another $C D N_{y}$ domain for the first time, the mobile trust degree of $u_{m}$ from $C D N_{x}$ to $C D N_{y}$ domain can be calculate as follows.

$$
\text { mobile_d } d_{u_{m}}^{C D N_{x} \rightarrow C D N_{y}}=\text { local_ } d_{u_{m}}^{C D N_{x}} \times d_{y x}^{C D N}
$$

Here, it should be noted that the trust degree between CDNs should use the one that $C D N_{y}$ rates $C D N_{x}$. And if $u_{m}$ already has the trust value (or trust degree) in $C D N_{y}$, the previous one is used continuously.

If $C D N_{y}$ is a "trust value" reputation system and the number of total participants is $m$, the mobile trust degree of $u_{m}$ can be transformed to the trust value in $C D N_{y}$ as follows.

$$
v_{u_{m}}^{C D N_{y}}=\text { mobile }_{-} d_{u_{m}}^{C D N_{x} \rightarrow C D N_{y}} \times \max _{j \in(1, m)} v_{j}^{C D N_{y}}
$$

And if $C D N_{y}$ is a "trust degree" reputation system, the mobile trust degree of $u_{m}$ can be considered as the trust degree in $C D N_{y}$ for all the other participants.

\section{Conclusion}

In this paper, we propose a hybrid CDNi-P2P architecture, and according to this architecture, we also propose two trust models: the local trust model and the cross-domain trust model. Based on the proposed trust models, the mobile user can transform his local trust to the mobile trust which can be taken to the new domain, 
and be converted to the new trust which can be used in the new domain. With the proposed models, we can avoid the disparate trust values of the same user in different domains and improve the availability of the contents possessed by the mobile users during the user moving among different domains. In the future, we will implement and assess the performance of our trust model in the hybrid CDNi-P2P network, and research on the available cross-domain trust models which can be used among different network architectures.

\section{Acknowledgements}

The work is supported by Basic Science Research Program through the National Research Foundation of Korea (NRF) funded by the Ministry of Education (2013R1A1A2011788). And it is also partially supported by the State Scholarship Fund organized by the China Scholarship Council (CSC).

\section{References}

[1] G. Pallis, A. Vakali, Insight and Perspectives for Content Delivery Networks. Communications of the ACM. 2006, 49 (1): 101-106.

[2] D. Xu, S. S. Kulkarni, C. Rosenberg, and H. K. Chai, Analysis of a CDN-P2P Hybrid Architecture for Cost Effective Streaming Media Distribution. Multimedia Systems. 2006, 11: 383-399.

[3] H. Yin, X. Liu, T. Zhan, V. Sekar, F. Qiu, C. Lin, H. Zhang, and B. Li, Design and deployment of a hybrid CDNP2P system for live video streaming: Experiences with LiveSky. Proc. of the 17th ACM international conference on Multimedia. 2009, pp. 25-34.

[4] L. Peterson, B. Davie, Framework for CDN Interconnection draft-ietf-cdni-framework-08. Internet draft in Network Working Group of Internet Engineering Task Force (IETF). 2014.

[5] David Hales, Bruce Edmonds, Applying a Socially Inspired Technique (Tags) to Improve Cooperation in P2P Networks. IEEE Trans. on Systems, Man, and Cybernetics-Part A: Systems and Humans. 2005, 35 (3): 385-395.

[6] G. Zacharia and P. Maes, Trust management through reputation mechanisms. Applied Artificial Intelligence. 2000, 14 (9): 881-908.

[7] M. Richardson, R. Agrawal, and P. Domingos, Trust management for the Semantic Web. Proc. of the Second International Semantic Web Conference. 2003, pp. 351-368.

[8] S.D. Kamvar, M.T. Schlosser, and H. Garcia-Molina, The EigenTrust algorithm for reputation management in P2P networks. Proc. of the Twelfth International World Wide Web Conference. 2003, pp. 1-12.

[9] S.D.Ramchurn, N.R.Jennings, C.Sierra, and L.Godo, A computational trust model for multi-agent interactions based on confidence and reputation. Proc.2nd Int. Joint Conf. on Autonomous Agents and Multiagent Systems (AAMAS), 2003, pp. 69-75.

[10] Y. Gil and V. Ratnakar, Trusting information sources one citizen at a time. Proc. of the first International Semantic Web Conference. 2000, pp. 162-176.

[11] eBay website. www.ebay.com.

[12] Amazon website. www.amazon.com.

[13] Taobao website. www.taobao.com. 